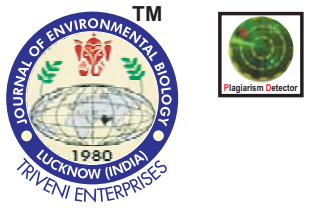

\title{
Establishing the botanical identity of plant drugs based on their active ingredients under diverse growth conditions
}

Authors Info

M. Iqbal ${ }^{1 *}$, Rabea Parveen ${ }^{2}$, Abida Parveen ${ }^{3}$, Bushra Parveen ${ }^{4}$ and I. M. Aref ${ }^{5}$

'Department of Botany, Jamia Hamdard,

New Delhi-10 062, India

2Department of Pharmaceutics, Jamia Hamdard,

New Delhi-110 062, India

${ }^{3}$ Faculty of Unani Medicine, Jamia Hamdard, New Delhi-110 062, India

${ }^{4}$ Department of Pharmacology, Jamia Hamdard,

New Delhi-110 062, India

${ }^{5}$ Department of Plant Production, College of Food \& Agricultural Sciences, King Saud University, Post Box 2460, Riyadh, 11451, Saudi Arabia

*Corresponding Author Email : iqbalg5@yahoo.co.in

Key words

Active ingredients

Environmental stress

Herbal drugs

Secondary metabolites

Indian medicine

\section{Publication Info}

Paper received : 06.02.2016

Revised received : 26.02.2017

Re-revised received : 28.04.2017

Accepted: 01.07.2017

\section{Abstract}

Higher plants are the major source of crude drugs used in herbal therapy. Adulteration in crudedrug material has necessitated scientific characterization of plant drugs, which is based primarily on morphological, anatomical and physico-chemical characters studied through microscopy and some analytical techniques. These criteria are helpful in isolating the adulterants from the actual drug, but fail to deliver when the very botanical identity of a plant drug is controversial, and several different plant species are used under a single drug name. This article suggests how to face this situation and overcome the problems likely to occur. Quantification of active ingredients in plant tissues is the only dependable way to identify the actual drug out of the different species in use. However, the amount of active ingredients in plants, responsible for the therapeutic efficacy of those plants, may vary with plant genotype and the habitat ecology, regulated largely by soil characters and agro-climatic conditions. Even within the individual plant, concentration of active ingredients often varies with plant parts/organs, stage of plant development and status of plant nutrition. Besides, degradation of environment may not only affect the quantity, but even the composition of the secondary metabolites produced. This situation may alter the chemical properties, and hence the degree of therapeutic effectiveness of these bioactive compounds. Thus, a careful estimation of active ingredients, taking

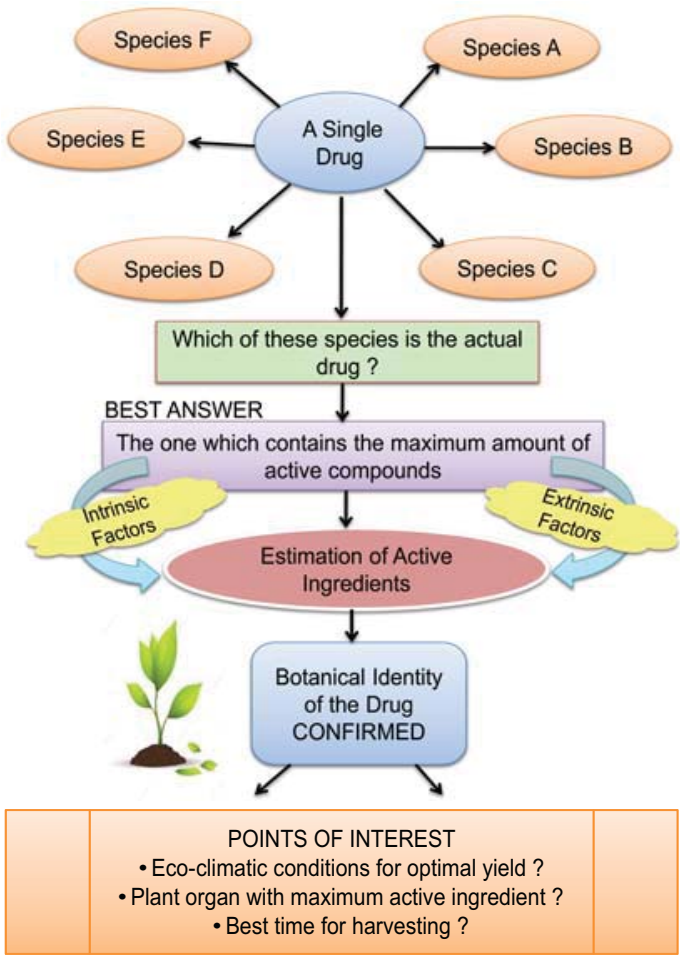
all the above-said situations into account, may decide which species should be regarded as the actual drug, which as an alternative drug and which as the non-drug (if the amount of the ingredient in question is just negligible). It may also indicate which plant organ should preferably be used and what should be the proper stage for harvesting. Further, environmental degradation may have a far-reaching influence on the therapeutic potential of medicinal plants and may also necessitate a re-fixing of drug doses, keeping in view the overall impact of environmental degradation on the active ingredients of these plants on one hand, and on the resistance level / immune system of human beings on the other. 


\section{Introduction}

According to 'The Plant List' (http://www.theplantlist.org), which supposedly contains all the known plant species and is maintained regularly by the Royal Botanic Garden and Missouri Botanical Garden, there are about 352,000 species of flowering plants coming from nearly 10500 genera that belong to 413 families (Steven, 2012). Many of these plants possess therapeutic properties and are frequently used to cure human as well as animal diseases. Crude drugs are obtained from a variety of higher plants. The majority of herbal drugs belong to the spermatophytes, particularly the angiosperms (flowering plants). Moreover, the number of plants known for their medicinal utility continues to increase with new folk claims consistently coming to light and being endorsed through pharmacognostic and pharmacological evaluations (Anis et al., 2000; Beigh et al., 2002, 2014; lqbal etal., 2011a).

\section{History of herbal medicine}

A cursory glance over the relevant literature reveals that the study of herbs dates back to nearly 5000 years. Sumerians, the people of Sumer, i.e. the southernmost region of the ancient Mesopotamia (the land between rivers Tigris and Euphrates, encompassing the southern Iraq and Kuwait of today), started dealing with herbal medicine in a formal way around 3000 years BC. Later, the art of healing developed in China, and the first Chinese herbal entitled 'Shennong Bencao', presenting a list of 365 medicinal plants with brief descriptions of their therapeutic importance, appeared as early as $2700 \mathrm{BC}$. In India, the use of herbal medicine began around 1900 BC. The ancient Indian herbalists, such as Charaka and Sushruta, described over 700 plants of medicinal significance. Egyptians started using plants for therapeutic purposes around 1000 years BC and the Arab scholars markedly contributed to the then existing knowledge of medical science (Beigh et al., 2002). During the 9th century AD, for instance, Al-Dinawari (828-896 AD) described some 637 plant drugs. Al-Qanoon Fit-Tib (The Canon of Medicine) of Abu Ali lbnSina (Avicenna; 980-1037 AD), referred to 800 tested drugs of plant and mineral origin. It was prescribed as a standard medical text at many medieval universities and remained in use till the middle of the 17th century. Recently, it was reprinted in New York in 1973. This book laid the foundation stone of clinical pharmacology, randomized controlled trials and efficacy tests, which gradually became the main stays of the modern medicine. Later, Ibn-al-Baitar (1197-1248 AD) described more than 1400 plants, including over 300 original discoveries, in the 13th century $A D$. All these works became the main source of information for subsequent publication of the European herbals (Pasquale, 1984).

The Indian systems of medicine : India has a long tradition of using drugs derived from plants. We now have three well established indigenous systems of medicine, namely Ayurveda, Unani and Siddha, of which the first two are based largely on herbs, whereas the last one has a greater focus on minerals. The Ayurvedic materia medica is a rich repository of herbs with a mention of about 2000 plant species (Beigh et al., 2002; Dar and Farooq, 1997). Vedic literature, especially Rigveda and Athrvaveda written sometime between 300 and 2000 BC, offered prescriptions for numerous maladies such as rheumatism, neuralgia, gout, jaundice, tumours, bronchitis, elephantiasis and skin diseases. The "Oshadi Suktam" of the Rigveda is perhaps the oldest scientific account of classification of medicinal plants. A systematic and detailed account of medicinal plants was later given in Charaka Samhita (1000-800 BC) by Charaka, known as the father of Ayurvedic medicine. Several popular drugs such as opium, hashish, dhatura, rauwolfia, nux-vomica, aconite, mustard seeds, lemon, antimony, sulphur, gold (considered as a potent drug), human milk, blood, animal testicles (especially of musk deer) were described by vernacular names in the VedicoBrahminic books of medicine, including Charaka Samhita and Sushruta Samhita (800-700 BC), the latter being the first document on Ayurvedic surgery (Pasquale, 1984; Raghunathan, 1987). In the recent history, Ayurveda flourished during the reign of Mughal emperor Akbar; it was in this period that Garcia D' Orta, a Portuguese physician and herbalist, published his 'Conversations on the simples, drugs and medicinal substances of India' in 1563, wherein he produced novel information on various medicinal plants and tropical human ailments (Shane, 2014). During the British rule, Indian therapies in general were suppressed and, alternatively, allopathy sprang up fast. British Pharmacopoeia was the most widely used compilation of western drugs those days. Ayurveda and other Indian therapies started regaining the ground around 1820 and became increasingly popular with the passage of time (Shane, 2014).

The emergence of Tamil language and Siddha system of medicine is believed to have been simultaneous in the Dravidian civilization, prior to the Aryan occupation of Sind region and Gangetic plain. Siddha is quite popular in Tamil Nadu and adjoining areas till date. Mythological believers connect it with Lord Shiva and his wife Parvati, who explained it to their son Lord Muruga, who transferred this knowledge to his disciple sage Agasthya (the father of Siddha medicine), who reportedly taught 18 Siddhars, who spread this knowledge into the human society. However, due to lack of supportive evidence, such claims have often been ignored by researchers in the field of medicine. The earlier epigraphic records in Tamil are said to have appeared in early part of the 7th century AD and Tholkappium, the oldest Tamil book, was written supposedly in the 11 th century. This book made a mention of Siddha medicine (Krishnamurthy and Mouli, 1984; Swamy, 1975). Siddha system identifies 4448 human diseases and its therapeutics is based mainly on metals and minerals.

Hippocrates (460-377 BC) laid the foundation of Unani therapy in Greece, which attained unprecedented heights by the efforts of Dioscorides ( $1^{\text {st }}$ century AD). His book, "De Materia Medica", remained a popular medical text for more than 1500 years during the Great Roman period and the middle ages. This medical science came to the Arab-Persian world in $800 \mathrm{AD}$, where it flourished optimally under the patronage of great scholars of that time such as Avicenna, Ibn Baithar and Rhazes. It came to 
India via the Central Asia around the $13^{\text {th }}$ century AD (Pasquale, 1984) and the Indian sub-continent became its biggest and final abode. It got exposed to modern scientific methodology in the $20^{\text {th }}$ century by the efforts of two eminent scholars, namely (a) Hakim Ajmal Khan (1868-1927), who initiated modern scientific research in Unani medicine with the help of Dr. Salimuzzaman Siddiqui FRS, who later isolated nine new alkaloids (viz. ajmaline, ajmalinine, ajmalicine, isoajmaline, neoajmaline, serpentine, serpentinine and two week bases with m.p. $220^{\circ}$ and $234^{\circ}$ ) from Asrol (Rauwolfia serpentina), and (b) Hakim Abdul Hameed (1908-1999), who founded Hamdard Laboratories (India) and also Jamia Hamdard, a first-grade university of medical, paramedical and allied sciences in New Delhi, and strengthened the Unani therapy by undertaking drug manufacture and clinical trials on modern scientific lines (Iqbal, 2011).

In addition, folk medicine is still popular in numerous tribal pockets throughout India, and extensively involves plant species. More than 4000 recognized ethnic groups constitute nearly $7.5 \%$ of the total Indian population. Despite having a great diversity of culture, food, habits, customs, and health-care concept, most of them use locally available herbal medicines to cure their common ailments. Nearly 19,000 species of vascular plants, 11,350 of nonvascular green plants and 14,500 of fungi are present in India (Jain, 2000). More than 2000 of them are reportedly used in ethnomedicine (Anonymous, 1994, 2000). A list of plants frequently used in the Indian systems of medicine for their important medicinal properties is given in Table 1.

Controversial botanical identity of herbal drugs : In ancient works, plant drugs were introduced normally by their general/vernacular names, and their descriptions were based mostly or solely on their morphological features. In the modern era, when old descriptions were matched with the morphology of similar-looking systematically classified and identified plant species, confusion arose in several cases due to overlapping of characters among different species. The botanical identity of many herbal drugs was also confused due to inconsistency in their vernacular/commercial names. There are instances where several plant species, often belonging to different genera and distant families, are known in the market by one and the same commercial name (Table 2a, 2b). Conversely, a single plant species is sometimes identified by different drug names in different regions. Use of various vernaculars in the indigenous literature often blurs the correct botanical identity of the raw materials. The number of plant drugs with controversial botanical identity now runs into hundreds. To determine a genuine species to represent a drug mentioned in the old scriptures, quantification of active metabolites of all the different species having a single vernacular name can be a dependable solution, and a comparison on this ground may distinguish the actual drug among so many claimants. Nonetheless, such an assessment involves certain intricacies and requires extreme precaution. In fact, the concentration of bioactive compounds may vary with plant genotype, agro-c1imatic condition, plant age, growth stage, and organ/part of the plant (Iqbal and Srivastava, 1998; Iqbal et al., 2011a). The nutritional and environmental factors may also have a bearing on quality as well as quantity of these ingredients (Arshi et al., 2006a, 2006b; Qureshi et al., 2013; lqbal et al., 2011b). The following text examines all these situations and presents an overview of recent efforts made to characterize the medicinal plants with especial reference to Indian perspectives.

\section{Intricacies of medicinal-plant characterization}

Researchers dealing with medicinal plants are often faced with a situation where plant collection is not authentic. Since the cultivation of medicinal plants is still very uncommon, more than $80 \%$ of their species are collected globally from the natural forests (Cordell, 2015). The samples so obtained may not be genuine due to deliberate or undeliberate adulteration or substitution. The plant collectors normally not being the trained plant taxonomists, may collect some other morphologically similar plant species, together with the actual one, due to carelessness or misidentification. Sometimes, adulteration can be done intentionally also; if the market price of the drug is high, the actual drug can be completely replaced by a similar-looking unconcerned but abundantly available cheaper plant material. However, in both cases, the actual drug species can be easily isolated from the adulterant(s) or fake substitutes on the basis of mutual comparison of their morphological, anatomical and physicochemical characters (Anonymous, 2007; Ahmad etal., 2014).

The medicinal plants have been characterized until recently on the basis of physical, structural, and phytochemical properties of the test material, with additional help from DNA fingerprinting and chromatography (Khan, 2011; Ahmad et al., 2014; Garg et al., 2014). Many modern techniques, concerning the molecular biology and proteomics, are now being used in research on medicinal plants (Chaudhary et al., 2014; Sarwat and Nabi, 2014; Mohsin et al., 2015). However, analysis of all these plant traits can help us in making the adulterants out only when the actual plant source of the drug is already known and the welldocumented information on its characters is available with us. In that case, using the relevant computer software, characters of the given drug sample can be compared, reliably and quickly, with those of the already identified genuine drug source. It takes no time in matching the plant signatures with utmost authenticity.

On the other hand, there are many herbal medicines whose botanical identity has been controversial due to lack of knowledge or inadequate information available. In such cases, several plant species are often used under a common drug name and the opinion of experts remains divided. In these cases, no morphological, structural, physico-chemical or molecular analysis of the given species can resolve the controversy, because here we are not sure which of the species should be treated as the 'standard' with whom the other candidates can be compared; thus there remains no room for character matching. In such a situation, quantification of plant metabolites of therapeutic importance present in all those species being used under a common drug name is the best and perhaps the only reliable 
approach (Iqbal et al., 2011a; lqbal 2013). The species containing the maximum of the compound concerned may be identified as the genuine drug, and those with lesser contents, as the possible alternatives. However, species having no or very little amount of that ingredient may be discarded and should no longer be used as a drug.

Plant metabolites : It is germane to clarify here that whereas the primary metabolites are the products of the anabolic and catabolic processes required for cell maintenance and proliferation, the secondary metabolites are chemical compounds that are not necessary for cell survival but contribute to plant fitness and survival in the environment. The secondary metabolites are identified mainly as: (a) N-containing metabolites, which are synthesized mostly from amino acids (e.g. alkaloids and glucosinolates), (b) non-nitrogenous metabolites, the phenolic compounds that have an aromatic ring substituted with a hydroxyl group (e.g. phenolic acids, coumarins, stilbenes, flavonoids tannins and lignins), and (c) terpenes, the derivatives of isoprenes composed almost entirely of carbon and hydrogen (e.g. plant volatiles, cardiac glycosides carotenoids and sterols). The structural diversity of most of these metabolites stems from the differentially modifying common backbone structures, with their derived compounds having divergent biological activities. Differential modification of the backbone structures has given rise to nearly 12000 known alkaloid structures and 10000 flavonoids with diverse biological activity (Kliebenstein, 2004; Ali, 2014). The most abundant secondary metabolites include terpenoids, phenylpropanoids, flavonoids and alkaloids (Ali, 2014). These are of great utility in the pharmaceutical and nutraceutical industry and offer cure for many human and livestock disorders (Beigh et al., 2002, 2003).

Many internal and external factors influence the plant chemistry and it is difficult to pinpoint a factor responsible for changes in chemical compounds. Several studies have shown the impact of normal environmental factors such as temperature, humidity and light, on production of secondary metabolites. Environmental stresses, like drought, salinity, heavy metals, a variety of other chemical pollutants and plant growth regulators have also shown diverse effects on biosynthesis of secondary metabolites (Ramakrishna \& Ravishankar 2012, Ali 2014). Normally, a mild stress has stimulatory effects (Selmar and Kleinwächter, 2013), this 'hormetic effect' effect has been discussed recently by Aref et al. (2016) while evaluating the impact of gamma radiations on the hyoscyamine production in Datura innoxia. However, strong stress is always inhibitive for plant metabolic activities.

\section{Factors affecting active ingredients}

As stated earlier, the fact remains that a realistic estimation of active ingredients in plant tissues is not always as simple as it is normally taken to be. Anumber of intrinsic as well as extrinsic factors, such as habitat topography, agro-climatic conditions, soil chemistry, cultivation techniques, genotypic variation, plant age, growth stage, organ-specific preferences and plant-nutrition level, often have a collective influence on the status of such compounds in plant tissues (Iqbal et al., 2011a; Dhir, 2015).

Habitat ecology and genotype factor : Studies carried out on artemisinin, a sesquiterpene active against the cerebral malaria, have shown that Artemisia annua plants growing in different regions of the world exhibit a remarkable effect of habitat ecology on their artemisinin content, which varies markedly with change in geographical/ecological conditions. It was 0.0 I to 0.09 per cent in different parts of India (Singh et al. 1986), 0.06 to 0.1 per cent in Argentina (Liersch et al. 1986), 0.01 to 0.5 per cent in different parts of China (Luo et al. 1980) and 0.06 to 0.22 per cent in the USA (Klayman et al. 1984, Ferreira et al. 1995). The compound was merely in traces in plants grown in Belgium, while its accumulation was maximum (about 0.42 and 0.50 ) in plants grown in Malaysia (Chan et al. 1997) and China (Luo et al. 1980), respectively. Some authors, however, consider it to be more a genotypic variation within a cross-pollinated population than a habitat-specific trait.

In a study of the bioactive compounds in Hypericum perforatum, high temperature and light intensity were found to have a positive influence on the accumulation of naphthodianthrones, acylphloroglucinol, hyperforin and phenolic compounds, with the highest level of most of these secondary metabolites occurring during the flowering phase (Radušiene et al., 2012). On the other hand, low temperature and light intensity caused a significant decline in the level of hypericins, which touched the lowest during the flowering and fruiting periods (Radušiene et al. 2012). However, it is often difficult to ascribe any observed change to some specific factor, because many abiotic factors usually cross-talk to a variety of internal factors and then a visible/perceptible impact sets in.

In a study of roots and rhizomes of anticancer plant Sinopodophyllum hexandrum, annual average precipitation, sunshine duration, soil $\mathrm{pH}$, soil organic matter and readily available $\mathrm{K}$ in the soil were found to influence the production of secondary metabolites, mainly podophyllotoxin, quercitin and kaempferol (Liu et al., 2015). The annual precipitation was significantly and negatively correlated to metabolite contents, whereas soil OM was the most important limiting factor and showed a positive and significant correlation with the production of metabolites. In general, climate factors were more effective than soil factors (Liu et al., 2015). A recent study has revealed a significant effect of the Mediterranean climate on the production of secondary metabolites in medicinal plants (Stavroula and Rahul, 2017)

Production of secondary metabolites in plants is often markedly affected by the local ecology (Ramakrishna and Ravishankar, 2012). Certain substances need specific environments for their production, while others may require them for enhanced synthesis. Alkaloid content of Aconitum 
Table 1 : Some common plant species (with their active ingredients) that are extensively used in India for therapeutic purposes

\begin{tabular}{|c|c|c|c|}
\hline Species & Part used & Active ingredient & Medicinal property \\
\hline $\begin{array}{l}\text { Aconitum } \\
\text { heterophyllum }\end{array}$ & Rhizome, Seeds & $\begin{array}{l}\text { Atesine, Aconitine, Hetidine, Hetisinone, } \\
\text { Heteratisine, Hetisine, Benzylleteratisine, } \\
\beta \text {-sitosterol, Carotene }\end{array}$ & $\begin{array}{l}\text { Aphrodisiac, Anti-rheumatic Antipyretic, } \\
\text { Astringent, Diuretic, Cure for Tonsillitis, } \\
\text { Cervical lymphadenitis }\end{array}$ \\
\hline Acorus calamus & Rhizome, Stem & $a$ and $\beta$-Asarone, Acoron, Eugenol & $\begin{array}{l}\text { Sedative, Laxative, Carminative, Diuretic, } \\
\text { Cure for Epilepsy and Depression }\end{array}$ \\
\hline Adhatoda vasica & $\begin{array}{l}\text { Leaves, Flowers, } \\
\text { Bark }\end{array}$ & $\begin{array}{l}\text { Vasicine, Vasicine acetate, Vasicinone, } \\
\text { Vasicinolone, 2-acetyle benzyle }\end{array}$ & $\begin{array}{l}\text { Expectorant, Relieve Cold, Cough, Asthma, } \\
\text { Whooping cough, Bronchitis }\end{array}$ \\
\hline Alcea rosea & $\begin{array}{l}\text { Roots, Flowers, } \\
\text { Seeds }\end{array}$ & $\begin{array}{l}\text { Flavonoids, Coumarins, Scopolitin, } \\
\text { Phytosterols, Asparagine, Tannins }\end{array}$ & $\begin{array}{l}\text { Diuretic, Astringent, Emollient, Demulcent, } \\
\text { Febrifuge, Anti-inflammatory, Cure for Goitre, } \\
\text { Urolithiosis, Ulceration }\end{array}$ \\
\hline Allium sativum & Bulbs & Allicin, Alliine, Alliinase, Inulin & $\begin{array}{l}\text { Antioxidant, Antitumor, Laxative, Antibiotic, } \\
\text { Antiviral, Antiseptic, Antiparasitic, Anti-cholestrol, } \\
\text { Aphrodisiac, Anti-diabetic, Thermogenic, Useful } \\
\text { in Baldness, Hair fall, Respiratory and } \\
\text { cardio-vascular diseases }\end{array}$ \\
\hline Aloe barbadensis & Succulent leaves & $\begin{array}{l}\text { Aloin, Emodin, Barbaloin, Isobarbaloin, } \\
\text { Aloetic acid, Anthranol, Neoalocin A, }\end{array}$ & $\begin{array}{l}\text { Purgative, Anti-inflammatory, Anti-UV-rays, } \\
\text { Skin protector, Humectant, Antimirobial, Cure Piles }\end{array}$ \\
\hline Ammi majus & Fruits & Xanthotoxin, Imperatorin, Bergapten & $\begin{array}{l}\text { Diuretic, Emmenagogue, Antihistosomal, } \\
\text { Cure for Psoriasis, Vitiligo, Leprosy }\end{array}$ \\
\hline $\begin{array}{l}\text { Arisaema } \\
\text { jacquemondii }\end{array}$ & Roots, Rhizome & Triterpenoids, Ariseminone & $\begin{array}{l}\text { Antimicrobial, Anthelmintic, Antioxidant, Useful } \\
\text { in Skin, Kidney, Menstrual \& Nervous disorders, } \\
\text { Toothache, Cough, Snake bite }\end{array}$ \\
\hline Artemisia annua & Whole plant & Artemisinin, Santonin & Anti-malarial, Anticancer, Antioxidant, Antiallergic \\
\hline Artemisia & Whole plant, especially & $\alpha$ and $\beta$-thujone, $p$-Cymene, 1,8-Cineole, & Anthementic, Nervine tonic, Stomachic, Febrifuge, \\
\hline absinthium & Leaves and Tops & Caryophyllene oxide, (Z)-Lanceol acetate & Cure for scurvy, abdominal disorders \\
\hline Azadirachta indica & All parts & $\begin{array}{l}\text { Nimbin, Nimbidin, Nimbolide, limonoids, } \\
\text { Quercitin, } \beta \text {-sitosterol }\end{array}$ & $\begin{array}{l}\text { Blood purifier, Anticancer, Anti-inflammatory, } \\
\text { Antifungal, Antibacterial, Antipyretic, Antiarthritic }\end{array}$ \\
\hline Bacopa monniera & Whole plant & $\begin{array}{l}\text { Brahmine, Herpestine, Apigenin, } \\
\text { Monnierasides, D-mannitol, Hersaponin } \\
\text { Cucurbitacin, Plantainoside B, Nicotine }\end{array}$ & $\begin{array}{l}\text { Nervine tonic, Cardiotonic, Mamory vitalizer, } \\
\text { Useful in Mental disorders, Diarrhoea, } \\
\text { Cataract, Bronchitis }\end{array}$ \\
\hline Butea & Bark, Seeds, & Butin, Isobutrin, Butein, Coreopsin, & Anti-hepatotoxic, Tonic, Anthelmintic, Diuretic, \\
\hline monosperma & Stem, Leaves & $\begin{array}{l}\text { Isocoreopsin, Monospermoside, } \\
\text { Isomonospermoside }\end{array}$ & Aphrodisiac, Purgative \\
\hline Camellia sinensis & Leaves & $\begin{array}{l}\text { Caffeine, Catechins, Proanthocyanidins, } \\
\text { Flavonols }\end{array}$ & $\begin{array}{l}\text { CNS stimulant, Bronchodilator, Cure for Asthma, } \\
\text { Angina pectoris, Coronary artery disease. }\end{array}$ \\
\hline Cassia angustifolia & Leaves & Sennosides, Aloe-emodin, Rhein & $\begin{array}{l}\text { Laxative, Purgative, Useful in Hepatomegaly, } \\
\text { Jaundice, Spleenomegaly, Anaemia }\end{array}$ \\
\hline Catharanthus & $\begin{array}{l}\text { Roots, Leaves, } \\
\text { Flowers }\end{array}$ & Vincristine, Vinblastine, Vincaleucoblastine, & Anti-cancer, anti-diabetic, anti-bacterial, \\
\hline & Flowers & $\begin{array}{l}\text { Veucocristine, AJmallcine, VIncelne, } \\
\text { Vineamine, Raubasin, Reserpine, } \\
\text { Catharanthine }\end{array}$ & anti-oxidant, anti-Ulcer \\
\hline Centaurea iberica & Leaves & $\begin{array}{l}\text { Flavones, Steroids, Sesquiterpene } \\
\text { lactones, Dimeric lignan glucoside }\end{array}$ & $\begin{array}{l}\text { Anti-inflammatory, Wound healing, Antidiabetic, } \\
\text { Cure for eczema, skin infections }\end{array}$ \\
\hline $\begin{array}{l}\text { Cephalis } \\
\text { ipecacuanha }\end{array}$ & Roots, Rhizomes & Emetine, Cephaeline, Protoemetine & $\begin{array}{l}\text { Emetic, Expectorant, Antidysenteric, Useful in } \\
\text { Cough, Bronchitis }\end{array}$ \\
\hline $\begin{array}{l}\text { Chrysanthemum } \\
\text { cinerariaefolium }\end{array}$ & Flowers & Pyrethrin & Insecticide, Anti-fungal, Anti-bacterial \\
\hline Cichorium intybus & Roots & $\begin{array}{l}\text { Inulin, Sesquiterpene lactones, Mannitol, } \\
\text { Caffeic-acid derivatives,Coumarins, } \\
\text { Hydroxycoumarins, Flavonoids, Alkaloids, } \\
\text { Terpenoids, Steroids, }\end{array}$ & $\begin{array}{l}\text { Antiallergic, Antineoplastic, Antihyperlipidemic, } \\
\text { Immunostimulator, Cure for Typhoid, Digestive } \\
\text { disorders }\end{array}$ \\
\hline $\begin{array}{l}\text { Cinchona } \\
\text { ledgeriana }\end{array}$ & Bark & $\begin{array}{l}\text { Quinine, Quinidine, Cinchonine, } \\
\text { Cinchonidine, Quinicine, Cinchonicine } \\
\text { Hydroquinine, Hydrocinchonidine } \\
\text { Homocinchonidine }\end{array}$ & Anti-malarial, Antipyretic, Stomachic \\
\hline Crocus sativus & Stigma, Upper & Protocrocin, Crocetin, Picrocrocin & Cosmetic, Tonic, Aphrodisiac, Sedative, \\
\hline
\end{tabular}




\begin{tabular}{|c|c|c|c|}
\hline & part of style & & Sposmolytic, Anti-cold \\
\hline Curcuma longa & Rhizome & $\begin{array}{l}\text { Curcumin, Demethoxycurcumin, } \\
\text { Bisdemethoxycurcumin, Termerone, } \\
\text { Atlantone, Zingiberene }\end{array}$ & $\begin{array}{l}\text { Antibiotic, Analgesic, Anti- inflammatory, } \\
\text { Antioxidant, Antitumour, Anti-amyloid, Blood } \\
\text { purifier }\end{array}$ \\
\hline Cymbopogon & Root, Leaves, & Citral, Geraniol, Apigenin, Citronella, & Antiseptic, Anti-mosquito, Stomachic, \\
\hline citratus & Whole plant & $\begin{array}{l}\text { Luteolin, Quercetin, Kaempferol, Caffeic } \\
\text { acid, Chlorogenic acid, p-Coumaric acid }\end{array}$ & $\begin{array}{l}\text { Emmenagogue, Diuretic, Cure for Toothache, } \\
\text { Headache, Neuralgia, Sprain }\end{array}$ \\
\hline Datura stramonium & $\begin{array}{l}\text { Flowering tops, } \\
\text { Leaves, Seeds }\end{array}$ & Hyoscyamine & Anti-asthmatic, Antirheumatic \\
\hline Daucus carota & Leaves, Seeds & $\begin{array}{l}\text { Carotol, Daucol, Daucucarotol, Copaenol, } \\
\text { Chromones, Sesquiterpenes, Flavonoids, } \\
\text { Coumarins, Anthcyanins }\end{array}$ & $\begin{array}{l}\text { Antifungal, Antibacterial, Hepatoprotective, } \\
\text { Anticancer, Antirheumatic, Cure for Eczema, } \\
\text { Ulceration, Amenorrhea, Toothache }\end{array}$ \\
\hline $\begin{array}{l}\text { Descurainia } \\
\text { sophia }\end{array}$ & Seeds, Leaves & $\begin{array}{l}\text { Analgesic, Antipyretic, Hypoglycemic, } \\
\text { Anti-inflammatory }\end{array}$ & $\begin{array}{l}\text { Quercitin, Descurainin, Descurainoside B, } \\
\text { Lactones, Kaempferol, Linolenic acid }\end{array}$ \\
\hline Digitalis lanata & Leaves & $\begin{array}{l}\text { Acetyldigoxin, Gitoxin, Digitoxin, } \\
\text { Digoxin, Lanatosides }\end{array}$ & Cardio-tonic, Diuretic \\
\hline Digitalis purpurea & Leaves & Gitoxin, Digitoxin & Useful in Cardio-vascular disorders, wound healing \\
\hline Dioscorea deltoidea & Tubers & Diosgenin, Corticosterone, Sigmasterol & $\begin{array}{l}\text { Oral contraceptive, Antifertility, Vermifuge, Useful } \\
\text { in cardio-vascular \& CNS disorders }\end{array}$ \\
\hline Ephedra sinica & Stem, Leaves & Ephedrine, Pseudoephedrine & $\begin{array}{l}\text { Stimulant, Thermogenic, Nasal and bronchial } \\
\text { decongestant }\end{array}$ \\
\hline Ficus carica & $\begin{array}{l}\text { Fruits, Stem and } \\
\text { Leaves }\end{array}$ & $\begin{array}{l}\text { Furocoumarins, Phytosterols, Flavonoids, } \\
\text { Anthocyanins, Catechins, Epi-catechins }\end{array}$ & $\begin{array}{l}\text { Antioxidant, Purgative, Anti-inflammatory, } \\
\text { Stomachic, Vermifuge, Relieves Skin viral } \\
\text { infections. Piles. Dvsentery. Diarrhoea }\end{array}$ \\
\hline Fragaria nubicola & Roots, Rhizome & Agrimoniin, Flavonoids & Menstrual disorder, Tonsilitis \\
\hline Glycyrrhiza glabra & Roots, Rhizomes & $\begin{array}{l}\text { Glycirrhizin, Glabranin A\&B, Glycyrrhetol, } \\
\text { Glabrolide, Isoflavones, Coumarins, } \\
\text { Triterpene }\end{array}$ & $\begin{array}{l}\text { Expectorant, Antiulcer, Hypocholesterolemic, } \\
\text { Anti-inflammatory, Antioxidant, Useful in } \\
\text { Pharyngitis, epilepsy, Anaemia, Haemorrhage, } \\
\text { Urticaria }\end{array}$ \\
\hline Hordeum vulgare & Grains, Leaves & $\begin{array}{l}\text { Saponarin, Saponaretin, Vitexin, } \\
\text { Lutonarin, lutonaretin, Lutaxin, Luteolin }\end{array}$ & $\begin{array}{l}\text { Antiurolithiatic, Antioxidant, Hypoglycemic, } \\
\text { Expectorant, Demulcent, Galactofuge, Lenitive, } \\
\text { Stomachic, Emollient, Febrifuge, Lowers blood } \\
\text { pressure, sugar \& Cholesterol \& body weight }\end{array}$ \\
\hline Hyoscyamus niger & $\begin{array}{l}\text { Flowering tops, } \\
\text { Leaves, }\end{array}$ & Hyoscine, Hyoscuarnine, Atropine & Useful in Asthma and Whooping cough; Purgative \\
\hline Iris kashmiriana & Rhizome, Bulb & $\begin{array}{l}\text { Flavonoids, Triterpenoids, Isoflavones, } \\
\text { Quinones }\end{array}$ & $\begin{array}{l}\text { Anti-inflammatory, Extrenal treatment for } \\
\text { Rheumatism, Eczema }\end{array}$ \\
\hline Lepidium sativum & $\begin{array}{l}\text { Seeds, Leaves, } \\
\text { Roots }\end{array}$ & $\begin{array}{l}\text { Imidazole, Lecidenes, Semilepidinosides, } \\
\beta \text {-carotene, Several Fatty acids }\end{array}$ & $\begin{array}{l}\text { Antihypertensive, Analgesic, Anti-inflammatory, } \\
\text { Depurative, Anticoagulant, Abortifacient, } \\
\text { Hypoglycemic, Antispasmodic, Aphrodisiac, } \\
\text { Galactagogue, Relieves Asthma, Irregular periods, } \\
\text { Hair loss, Iron deficiency }\end{array}$ \\
\hline Malva neglecta & $\begin{array}{l}\text { Stem, Petiole, } \\
\text { Leaf, Fruit }\end{array}$ & $\begin{array}{l}\text { Phenols, Favonoids, Hydrocinnamic } \\
\text { acids, Anthocyanin, Proanthocyanidin }\end{array}$ & $\begin{array}{l}\text { Cure for Urinary \& Respiratory inflammations, } \\
\text { Kidney stone, Haemorrhoids, Menstrual disorder, } \\
\text { Abdominal pain, Diabetes }\end{array}$ \\
\hline Mentha arvensis & Leaves, Flowers & Menthol & Rubefacient, Carminative, Anti- spasmodic \\
\hline $\begin{array}{l}\text { Moringa } \\
\text { pterygosperma }\end{array}$ & All plant parts & $\begin{array}{l}\text { Anthomine, Moringinine, Moringyne, } \\
\text { Pterigospermin, Spirochine, } \\
\text { Thicarbamates, Benzilisothio-cyanates }\end{array}$ & $\begin{array}{l}\text { Stimulant, Aphrodisiac, Antispasmodic, Anti- } \\
\text { hypotensive, Antiepileptic, Antihysteric, Antipalsy, } \\
\text { Anti-rheumatic, Antiascitic, Anti-inflammatory, } \\
\text { Diuretic }\end{array}$ \\
\hline Narcissus tazetta & Bulbs & Phytoene, Phytofluene & External application to cure boils \\
\hline Nymphea alba & Leaves and fruits & Nupharine, Nymphaeine & External application to cure boils \\
\hline $\begin{array}{l}\text { Papaver } \\
\text { somniferum }\end{array}$ & $\begin{array}{l}\text { Fruits, Capsules, } \\
\text { Leaves }\end{array}$ & $\begin{array}{l}\text { Codein, Morphine, Narcotine, Papaverine, } \\
\text { Noscapine, Thebaine }\end{array}$ & $\begin{array}{l}\text { Analgesic, Antidiarrhoeal, Muscle relaxant, Cures } \\
\text { Cough, Sneezing, Irregular breathing }\end{array}$ \\
\hline Picrorhiza kurroa & Rhizomes & Picroside, Kutkoside, Apocyanine & $\begin{array}{l}\text { Bitter tonic, Stomachic, Febrifuge, Anti-malarial, } \\
\text { Cathartic, Hepatotoxic, Cholagogue }\end{array}$ \\
\hline Plantago indica & Seeds & $\begin{array}{l}\text { Xylin, Mucilage polysaccharide, Anvrtyn, } \\
\text { Aucubin, Plantagin, Adenine Choline, } \\
\text { Aeocoeine }\end{array}$ & $\begin{array}{l}\text { Cathartic, Purgative, Useful in Constipation, } \\
\text { Humorrhoids, Hypercholesterolemia }\end{array}$ \\
\hline
\end{tabular}




\begin{tabular}{|c|c|c|c|}
\hline $\begin{array}{l}\text { Plantago } \\
\text { lanceolata }\end{array}$ & Leaves & $\begin{array}{l}\text { Aucubin, Catalpol, Lavandulifolioside, } \\
\text { Plantamajoside, Acteoside, Cistanoside }\end{array}$ & $\begin{array}{l}\text { Blood purifier, Cure for cough, dermal and } \\
\text { respiratory tract disorders }\end{array}$ \\
\hline $\begin{array}{l}\text { Podophyllum } \\
\text { hexandrum }\end{array}$ & Rhizomes & Podophyllotoxin, Podophylloresin & Anti-cancerous, Anti-HIV \\
\hline Potentilla fruticosa & Leaves & Rutin, Flavonoids, Phenols & Cure for Diarrhoea, Hepatitis, Rheuma, Scabies \\
\hline Punica granatum & Exocarp, Fruit & $\begin{array}{l}\text { Ellagic acid, Punicic acid, Punicalagins, } \\
\text { Anthocyanins, Anthocyanidins }\end{array}$ & $\begin{array}{l}\text { Wound healer, Antiparasitic, Antiulcer, Anti- } \\
\text { inflammatory, Antiatherogenic, Hypoglycemic, } \\
\text { Cure for diarrhoea and bronchitis }\end{array}$ \\
\hline $\begin{array}{l}\text { Ranunculus } \\
\text { arvensis }\end{array}$ & Leaves & $\begin{array}{l}\text { Protoanemonin and its precursor } \\
\text { Ranunculin }\end{array}$ & Antifungal, Cure for eczema, asthma, fevers, gouts \\
\hline $\begin{array}{l}\text { Rauwolfia } \\
\text { serpentina }\end{array}$ & Roots & $\begin{array}{l}\text { Serpentine, Reserpine, Ajmalicine, } \\
\text { Rescinamone }\end{array}$ & $\begin{array}{l}\text { Anti-hepatotoxic, Cure for snake bite and mental } \\
\text { disorders }\end{array}$ \\
\hline $\begin{array}{l}\text { Rhamnus } \\
\text { purshiana }\end{array}$ & Bark & Cascarosides, Emodin & $\begin{array}{l}\text { Purgative, Bitter tonic, Stimulant laxative, } \\
\text { Anticancer }\end{array}$ \\
\hline Rheum emodi & Rhizome & $\begin{array}{l}\text { Emodin, Rhein, Physcion, Sennosides } \\
\text { A\&B,Picetannol, Resveratrol }\end{array}$ & $\begin{array}{l}\text { Hepato- and nephroprotective, Antioxidant, anti- } \\
\text { inflammatory, Antidislipidemic, Antidiabetic, } \\
\text { Antimicrobial, Purgative, Immunoenhancer }\end{array}$ \\
\hline Saussurea lappa & Roots & $\begin{array}{l}\text { Saussurine, Inulin, Potassium nitrate, } \\
\text { Kushtin }\end{array}$ & $\begin{array}{l}\text { Cardiac stimulant, Anti-septic, Anti-infectant, } \\
\text { Spasmolytic, Antirheumatic }\end{array}$ \\
\hline Silybum marianum & Roots, Seeds & Silybin, Silymarin, Silydianin & $\begin{array}{l}\text { Treatment of liver disorders, Jaundice and Gall } \\
\text { stone }\end{array}$ \\
\hline $\begin{array}{l}\text { Sinopodophyllum } \\
\text { hexandrum }\end{array}$ & Roots, Rhizomes & Podophyllotoxin, Quercitin, Kaempferol & $\begin{array}{l}\text { Anticancer, Antiarthritis, Anti-inflammatory, } \\
\text { Cardiotonic }\end{array}$ \\
\hline Solanum nigrum & $\begin{array}{l}\text { Fruits, Seeds, } \\
\text { Leaves }\end{array}$ & $\begin{array}{l}\text { Solanine, Solasodine, Solanigrosides, } \\
\text { Systenin,Tigogenin }\end{array}$ & $\begin{array}{l}\text { Antioxidant, Anticonvulsant, Antipyretic, } \\
\text { Hepatoprotective, Immunomodulator, Laxative, } \\
\text { Sedative, Antiasthmatic }\end{array}$ \\
\hline $\begin{array}{l}\text { Strychnos } \\
\text { nuxvomica }\end{array}$ & Seeds, Bark & Strychnine, Brucine, Loganine & $\begin{array}{l}\text { CNS stimulant, Respiratory stimulant, Antipalsy, } \\
\text { Relieves facial paralvsis. }\end{array}$ \\
\hline Taxus baccata & Stem & Taxine, Taxicantin, Baccatin, Ephedrine & $\begin{array}{l}\text { Anti-cancer, Antitussive, Abortive, Emenegogue, } \\
\text { Diuretic, Laxative }\end{array}$ \\
\hline Tithonia diversifolia & All parts & $\begin{array}{l}\text { Sesquiterpene lactones, Caffeoylquinic } \\
\text { acid }\end{array}$ & $\begin{array}{l}\text { Antimalarial, Antimicrobial, Antihyperglycemic, } \\
\text { Gastro-protective }\end{array}$ \\
\hline Tribulus terrestris & Leaves, Fruits & $\begin{array}{l}\text { Flavonols, Flavonol glycosides, Steroidal } \\
\text { saponins, Alkaloids }\end{array}$ & $\begin{array}{l}\text { Antidiabetic, Antispasmodic, Anticariogenic, } \\
\text { Cardiotonic, Aphrodisiac, Analgesic, } \\
\text { Immunomodulatory, Diuretic, Libido promoter, } \\
\text { Useful in CNS disorders, Gall stones and } \\
\text { Erectile dysfunction }\end{array}$ \\
\hline Viburnum & Root, Stem, & Alkaloids, Flavonoids, Steroids, & Antioxidant, Antimicrobial, Antiseptic, \\
\hline grandiflorum & $\begin{array}{l}\text { Leaves, Bark, } \\
\text { Seeds }\end{array}$ & $\begin{array}{l}\text { Terpenoids, Vibsane diterpenoid, } \\
\text { Anthraquinone, Saponins, Triterpene, } \\
\text { Butilinol, Butelene, Alpha-amyrin }\end{array}$ & $\begin{array}{l}\text { Antinociceptive, Anticarcinomic, Antirheumatic, } \\
\text { Antimicrobial, Cure for Malaria, Whooping } \\
\text { cough, Liver disorders }\end{array}$ \\
\hline Viola odorata & Leaves and Flowers & Volatile compounds, Friedelin, $\beta$-sitosterol & $\begin{array}{l}\text { Expectorant, Antibronchitis, Cure for cold \& cough, } \\
\text { skin and pulmonary disorders, insomnia, }\end{array}$ \\
\hline Withania somnifera & Roots, Stem & $\begin{array}{l}\text { Somnine, Somniferine, Anferine, } \\
\text { Flavonoids, Lactones, Acyl steryl } \\
\text { glucosides, Withanolides }\end{array}$ & Anti-ulceration, Anti-anxiety, Anticholesterol \\
\hline Ziziphus jujuba & $\begin{array}{l}\text { Seeds, Bark, } \\
\text { Fruits, Leaves }\end{array}$ & $\begin{array}{l}\text { Zizogenin,Ziziphin,Spinocin, Swertish, } \\
\text { Colubrinic acid, Alphitolic acid, } \\
\text { Ziziberenelic acid, Betulinic acid }\end{array}$ & $\begin{array}{l}\text { Antipyretic, Aphrodisiac, Anxiolytic, Laxative, To } \\
\text { cure Jaundice, Gastric disorders, Urinary } \\
\text { infections, Cough, Headache, Leucorrhoea }\end{array}$ \\
\hline
\end{tabular}

Based largely on lqbal \& Srivastava (1998), Mughal et al. (1999) and Beigh et al. (2002; 2011a)

heterophyllum increased with increase in altitude; and among the different plant parts, tubers had the maximum (Beigh et al., 2005, 2008). Similar variations were observed for podophyllotoxin content in Podophyllum hexandrum (Iqbal et al., 2004). The increase in alkaloid production with rising altitude could be related to biochemical adaptation of the species in a given ecological, edaphic or climatic niche. It is known that, outside their normal ecological range or under disturbed ecosystem conditions, many medicinal plants behave atypically (Iqbal and Srivastava, 1998; Beigh et al. 2008). The marked decline observed in the morphological, physiological and biochemical traits of $A$. heterophyllum grown at a lower altitude could be due to this factor. Similarly, altitude was positively and significantly correlated to the contents of total phenolics, flavonoids and rutin, but showed a 
Table 2 a : Certain famous examples of plant drugs with controversial botanical identity, where several species of one or different genera are used as one and the same drug

\begin{tabular}{|c|c|c|c|c|c|}
\hline $\begin{array}{l}\text { Common/ } \\
\text { Commercial name }\end{array}$ & Botanical name & Family & $\begin{array}{l}\text { Common/ } \\
\text { Commercial name }\end{array}$ & Botanical name & Family \\
\hline Agnimath & $\begin{array}{l}\text { Clerodendron phlomides } \\
\text { C. indicum } \\
\text { C. infortunatum } \\
\text { Premna obtusifolia } \\
\text { P. serratifolia }\end{array}$ & $\begin{array}{l}\text { Verbenaceae } \\
\text {-do- } \\
\text {-do- } \\
\text {-do- } \\
\text {-do- }\end{array}$ & $\begin{array}{l}\text { Brahmi } \\
\text { Gaozaban }\end{array}$ & $\begin{array}{l}\text { Premna herbacea } \\
\text { Bacopa monniera } \\
\text { Centella asiatica } \\
\text { Anisomelis indica } \\
\text { Arnebia benthamii }\end{array}$ & $\begin{array}{l}\text { Verbenaceae } \\
\text { Scrophulariaceae } \\
\text { Apiaceae } \\
\text { Lamiaceae } \\
\text { Boraginaceae }\end{array}$ \\
\hline Amla Veta & $\begin{array}{l}\text { Ampelocissus latifolia } \\
\text { Cayratia trifolia } \\
\text { Cissus repens } \\
\text { C. vitiginea } \\
\text { Garcinia pedunculata } \\
\text { Rheum australe } \\
\text { Rumex vesicarius }\end{array}$ & $\begin{array}{l}\text { Vitaceae } \\
\text {-do- } \\
\text {-do- } \\
\text {-do- } \\
\text { Clusiaceae } \\
\text { Polygonaceae } \\
\text {-do- }\end{array}$ & & $\begin{array}{l}\text { Coccinia glauca } \\
\text { Elephantopus scaber } \\
\text { Heliotropium } \\
\text { ophioglossum } \\
\text { Onosma bracteatum } \\
\text { Trichodesma indicum } \\
\text { T. zevlanicum }\end{array}$ & $\begin{array}{l}\text {-do- } \\
\text { Asteraceae } \\
\text { Boraginaceae } \\
\text {-do- } \\
\text {-do- } \\
\text {-do- }\end{array}$ \\
\hline Arjuna & $\begin{array}{l}\text { Kavalama urens } \\
\text { Lagerstromia peciosa } \\
\text { Terminalia arjuna } \\
\text { T. tomentosa }\end{array}$ & $\begin{array}{l}\text { Sterculiaceae } \\
\text { Lythraceae } \\
\text { Combretaceae } \\
\text {-do- }\end{array}$ & Jivanti & $\begin{array}{l}\text { Cimicifuga foetida } \\
\text { Dregia volubilis } \\
\text { Flickingeria nodosa } \\
\text { Gymnema svlvestris }\end{array}$ & $\begin{array}{l}\text { Ranunculaceae } \\
\text { Asclepiadaceae } \\
\text { Orchidaceae } \\
\text { Asclepiadaceae }\end{array}$ \\
\hline Ashok & $\begin{array}{l}\text { Bauhinia purpurea } \\
\text { B. variegata } \\
\text { Polyalthia longifolia } \\
\text { Saraca asoca } \\
\text { Shorea robusta } \\
\text { Trema orientalis }\end{array}$ & $\begin{array}{l}\text { Caeaslpiniaceae } \\
\text {-do- } \\
\text { Fabaceae } \\
\text { Fabaceae } \\
\text { Dipterocarpaceae } \\
\text { Ulmaceae }\end{array}$ & Laxamana & $\begin{array}{l}\text { Holostemma adakodien } \\
\text { Leptadenia reticulata } \\
\text { Sarcostemma acidum } \\
\text { Trema orientalis } \\
\text { Atropa mandragora } \\
\text { Biophytum sensitivum }\end{array}$ & $\begin{array}{l}\text {-do- } \\
\text {-do- } \\
\text {-do- } \\
\text { Urticaceae } \\
\text { Solanaceae } \\
\text { Oxalidaceae }\end{array}$ \\
\hline Bharangi & $\begin{array}{l}\text { Clerodendron serratum } \\
\text { C. indicum } \\
\text { Ceriscoides turgida } \\
\text { Picrasma quassioides }\end{array}$ & $\begin{array}{l}\text { Verbenaceae } \\
\text {-do- } \\
\text { Rubiaceae } \\
\text { Simaroubaceae }\end{array}$ & & $\begin{array}{l}\text { Ipomoea sepiaria } \\
\text { Mandragora autumnalis } \\
\text { Smithia geminifera }\end{array}$ & $\begin{array}{l}\text { Convolvulaceae } \\
\text { Solanaceae } \\
\text { Papilionaceae }\end{array}$ \\
\hline
\end{tabular}

negative relationship with the content of tannins in Potentilla fruticosa (Liu et al., 2016). Annual sunshine also had a positive effect on flavonoids and rutin contents. Annual mean temperature showed a negative correlation with the accumulation of total phenolics (Liu et al., 2016). Likewise, altitude and annual mean temperature were significantly and positively correlated to the contents of chlorogenic acid and flavonoids in Eucommia ulmoides (Dong et al., 2011); further, the geniposidic acid content showed a significant and positive relationship with the annual sunshine duration but a significant and negative correlation with the annual mean temperature (Dong et al., 2011).

Biosynthesis of secondary metabolites in these species, despite being a genetically controlled phenomenon, seems to be greatly influenced by both the biotic and abiotic environmental factors. Sometimes hormesis may also be operative, wherein mild stress acts as a stimulant for the metabolic activities of the biological system exposed and, consequently, the metabolite production is enhanced (Aref et al., 2016). Water stress, radiation impact and other stresses in mild dose often act as elicitors and enhance metabolite production (Dhir, 2015). Increased accumulation of carbohydrate is often a signal of enhanced production of secondary metabolites, which would add to the medicinal quality of the plant (Al-Gabbiesh et al., 2015). It is important to initiate studies aimed at identifying conditions that are optimal for plant growth and for the formation of active secondary metabolites, and then undertake programs of selection and genetic upgrading to conserve the endangered medicinal plants and develop suitable cultivars for their largescale cultivation.

In the case of a medicinal plant species having several genotypes, the level of active principle in plant tissues/organs may also exhibit genotypic variation. For instance, Centella asiatica, a memory-vitalizing plant drug of the family Apiaceae, contains asiaticoside and madecassoside as its main active compounds. In its genotype F-line (with heavily-fringed leaf margin), the content of asiaticoside was almost half the content of madecassoside, whereas the ratio was just reverse in the genotype S-line (with smooth leaf margin) (Aziz et al., 2007).

Culture media, growth stage and plant parts : In a study of Artemisia annua, artemisinin was not detectable in the in-vitro produced callus; a negligible amount $(0.004-0.006 \%)$ was detected in multiple-shoot regenerants, but it was considerably large in pre-flowering stage $(0.08-0.10 \%)$ and flowering stage $(0.10-0.12 \%)$ of plantlets grown in vitro (Gulati et al., 1996). Similar variations of artemisinin content in relation to stages of 
Table $2 \mathbf{b}$ : Certain famous examples of plant drugs with controversial botanical identity, where several species of one or different genera are used as one and the same drug

\begin{tabular}{|c|c|c|c|c|c|}
\hline $\begin{array}{l}\text { Common/ } \\
\text { Commercial name }\end{array}$ & Botanical name & Family & $\begin{array}{l}\text { Common/ } \\
\text { Commercial name }\end{array}$ & Botanical name & Family \\
\hline Pashanbheda & $\begin{array}{l}\text { Aerva lanata } \\
\text { Ammannia baccifera } \\
\text { Bryophyllum pinnatum } \\
\text { Homonoia riparia } \\
\text { Plectranthus amboinicus } \\
\text { Rotula aquatica }\end{array}$ & $\begin{array}{l}\text { Amaranthaceae } \\
\text { Lythraceae } \\
\text { Crassulaceae } \\
\text { Euphoribaceae } \\
\text { Lamiaceae } \\
\text { Boraginaceae }\end{array}$ & $\begin{array}{l}\text { Rudanti } \\
\text { Sariva }\end{array}$ & $\begin{array}{l}\text { Potentilla nepalensis } \\
\text { Capparis moonii } \\
\text { Cressa cretica } \\
\text { Cryptolepis buchananii } \\
\text { Decalepis hamiltoni } \\
\text { Hemidesmus indicus }\end{array}$ & $\begin{array}{l}\text { Rosaceae } \\
\text { Capparaceae } \\
\text { Convolvulaceae } \\
\text { Asclepiadaceae } \\
\text { Apocynaceae } \\
\text { Asclepiadaceae }\end{array}$ \\
\hline Patha & $\begin{array}{l}\text { Cissampelos pareira } \\
\text { Cyclea peltata } \\
\text { Stephania japonica }\end{array}$ & $\begin{array}{l}\text { Menispermaceae } \\
\text {-do- } \\
\text {-do- }\end{array}$ & Shankhpushpi & $\begin{array}{l}\text { Ichnocarpus frutescens } \\
\text { Canscora diffusa } \\
\text { Clitoria ternatea }\end{array}$ & $\begin{array}{l}\text { Apocynaceae } \\
\text { Gentianaceae } \\
\text { Papilionaceae }\end{array}$ \\
\hline Prisniparni & $\begin{array}{l}\text { Desmodium gangeticum } \\
\text { D. laxiflorum } \\
\text { D. velutinum } \\
\text { Uraria lagopoides } \\
\text { U. picta }\end{array}$ & $\begin{array}{l}\text { Fabaceae } \\
\text {-do- } \\
\text {-do- } \\
\text {-do- } \\
\text {-do- }\end{array}$ & Talis Patra & $\begin{array}{l}\text { Convolvulus microphyllus } \\
\text { Evolvulus alsinoides } \\
\text { Lavendula bipinnata } \\
\text { Abies spectabilis } \\
\text { Flacourtia jangomas }\end{array}$ & $\begin{array}{l}\text { Convolvulaceae } \\
\text {-do- } \\
\text { Lamiaceae } \\
\text { Pinaceae } \\
\text { Salicaceae }\end{array}$ \\
\hline Punarnava & $\begin{array}{l}\text { Boerhaavia diffusa } \\
\text { Trianthema } \\
\text { portulacastrum }\end{array}$ & $\begin{array}{l}\text { Nyctaginaceae } \\
\text { Aizoaceae }\end{array}$ & & $\begin{array}{l}\text { Rhododendron } \\
\text { anthopogon } \\
\text { Taxus baccata }\end{array}$ & $\begin{array}{l}\text { Ericaceae } \\
\text { Taxaceae }\end{array}$ \\
\hline Rasna & $\begin{array}{l}\text { Alpinia galanga } \\
\text { Aristolochia indica } \\
\text { Enicostemma littorale } \\
\text { Pluchea lanceolata } \\
\text { Vanda tessellate } \\
\text { Viscum album } \\
\text { Withania coaqulans }\end{array}$ & $\begin{array}{l}\text { Zingiberaceae } \\
\text { Aristolochiaceae } \\
\text { Gentianaceae } \\
\text { Asteraceae } \\
\text { Orchidaceae } \\
\text { Loraanthaceae } \\
\text { Apocynaceae }\end{array}$ & Vatsanabh & $\begin{array}{l}\text { Aconitum chasmanthum } \\
\text { A. ferox } \\
\text { A. heterophyllum } \\
\text { A. napellus } \\
\text { Coptis teeta } \\
\text { Delphinium denudatum }\end{array}$ & $\begin{array}{l}\text { Ranunculaceae } \\
\text {-do- } \\
\text {-do- } \\
\text {-do- } \\
\text {-do- } \\
\text {-do- }\end{array}$ \\
\hline Ratanjot & $\begin{array}{l}\text { Withania coagulans } \\
\text { Alkanna tinctoria } \\
\text { Anemone obtusiloba } \\
\text { Geraneum wallichianum } \\
\text { Jatropha glandulifera } \\
\text { Onosma echioides }\end{array}$ & $\begin{array}{l}\text { Apocynaceae } \\
\text { Boraginaceae } \\
\text { Ranunculaceae } \\
\text { Geraniaceae } \\
\text { Euphorbiaceae } \\
\text { Boraginaceae }\end{array}$ & Vriddha Daru & $\begin{array}{l}\text { Marsdenia tenacissima } \\
\text { Merremia peltata } \\
\text { Operculina turpethum } \\
\text { Salvia plebeia } \\
\text { Thespesia populnea }\end{array}$ & $\begin{array}{l}\text { Asclepiadaceae } \\
\text { Convolvulaceae } \\
\text {-do- } \\
\text { Lamiaceae } \\
\text { Malvaceae }\end{array}$ \\
\hline
\end{tabular}

plant growth were noticed in plants grown in vivo. Likewise, the amounts of peruvoside and neriifolian, the well-known cardiotonics, were more in the callus than in the regenerants of Thevetia neriifolia (Apocynaceae). Plants grown in vivo displayed an intermediate status. Peruvoside production was more in cotyledonary leaf callus than in hypocotyl callus, but neriifolian content was similar in both these calli (Gulati et al., 2000).

Similarly, the amounts of vincristine and vinblastine, the famous cardenolides with anticancer activity, varied in different developmental stages of Catharanthus roseus cultures (Datta and Srivastava, 1997). Vincristine production was less (up to $0.5 \mu \mathrm{g} \mathrm{g}^{-1}$ dry weight) in the callus than in regenerants $\left(1.5 \mu \mathrm{g} \mathrm{g}^{-1}\right.$ dry weight). Similarly, vinblastine was noted to be merely $2.5 \mu \mathrm{g} \mathrm{g}^{-1}$ $\mathrm{dw}$ in the callus, but $9.2 \mathrm{\mu g} \mathrm{g}^{-1} \mathrm{dw}$ in regenerants. In the in-vivogrown plant material, vincristine content was comparable to that in the callus, whereas vinblastine content was comparable to that in regenerants. Nature of the medium also had an impact; the yield was relatively less on liquid medium than on solid medium (Datta and Srivastava, 1997).

Accumulation of hyoscyamine, a tropane alkaloid used in treating asthma, was much less in the in-vitro-raised callus or plantlets than in the field-grown plants of Datura innoxia. In the latter case, hyoscyamine content was relatively less $\left(0.80 \mathrm{mg} \mathrm{g}^{-1} \mathrm{dw}\right)$ in young leaves, more $\left(1.50 \mathrm{mg} \mathrm{g}^{-1} \mathrm{dw}\right)$ in old leaves and the maximum $\left(1.76 \mathrm{mg} \mathrm{g}^{-1} \mathrm{dw}\right.$ ) in medium-sized leaves, thus showing differences in relation to growth medium as well as the leaf age (Srivastava et al., 1993). Concentration of xanthotoxin, a furanocoumarin active against leucoderma and other dermal diseases, differed markedly in the field-grown and in-vitro-grown Ammi majus plants. It was only $4.0 \mathrm{mg} \mathrm{g}^{-1} \mathrm{dw}$ in leaves and $4.2 \mathrm{mg} \mathrm{g}^{-1} \mathrm{dw}$ in seeds of field-grown plants, but as high as $10.6 \mathrm{mg} \mathrm{g}^{-1} \mathrm{dw}$ in the in-vitro-raised regenerating callus (Purohit etal., 1995a, 1995b).

A comparative study of Valeriana officinalis cultivation in an aeroponic, floating medium (a perlite and vermiculite mix), and in soil systems, revealed a $57 \%$ enhancement in the boronyl acetate concentration in the floating system, compared with the soil system (Tabatabei, 2008). Many other crops also gave a superior yield in aeroponic cultivation, compared with the conventional field production (Pagilarulo et al., 2005; Hayden, 2006). Osmotic stress may have a bearing on the composition of essential oils. In fact, any factor that inhibits plant growth, can render more carbon available for the synthesis of secondary metabolites (Tabatabei, 2008). 
Psoralen, another furanocoumarin, shows activity against leucoderma and other dermal disorders. Its concentration varies not only in different plant parts/organs of Psoralea corylifolia but even within the organ, depending on the stage of plant growth. The psoralen content was highest $(5.32 \%)$ in seeds, much less (about $1 \%$ or less) in leaves, still less $(0.32-0.54 \%$ ) in the stem and the least $(0.28-0.34 \%)$ in roots. Moreover, its concentration in all these plant parts varied at the pre-flowering, flowering and post-flowering stages, being the highest during flowering and lowest in the post-flowering phase (Ali et al., 2008). Among the various plant parts of Aconitum heterophyllum, a highaltitude plant, tubers had the highest alkaloid contents, while the aerial stem contained the least showing the order of decline as: tubers > leaves > flowers > stem. In an ex-situ population at a lower altitude, however, the stem was richer in alkaloid content than flowers, depicting an order of $T>L>S>F$ (Beigh et al., 2008).

The above examples clearly demonstrate that production of active ingredients is markedly affected by the mode of cultivation and also varies with organs, plant age and growth stage. All these studies, together with many others, suggest that the estimation of active ingredients based on samples collected at any one stage of plant development or from any one organ/part of the plant or from a single habitat may not necessarily depict the actual status of the compound in the given species and, therefore, may not be realistic and fairly authentic.

Environmental pollutants : Environmental degradation is now an added factor that affects almost every walk of life in the modern world, including the health of living beings. A variety of environmental pollutants, including gases, particulates, heavy metals, chemicals, noise, and radiations, act as stressors in the atmosphere and affect activities in living systems. Sustained environmental pollutions normally increase the atmospheric temperature of plant habitats, causing water stress and often creating oxidative stress within plant tissues, which has a bearing on plant metabolic activities. The much talked-about 'global warming', which is supposed to cause retreat of glaciers, rise of sea level, frequent extreme weather events, increased water acidification, community migration, species extinction, altered crop yields, and human-health hazards, is also an outcome of the environmental degradation. Surprisingly, the altered efficacy of medicinal plants has hardly been discussed and evaluated seriously in relation to the environmental pollution that has caused a variety of stresses.

It is now well understood that environmental stresses have a potential to alter the quantity, and sometimes even the quality, of secondary metabolites (Faroogi and Haque, 2011; Iqbal et al., 2011b; Katare and Bora, 2011). Various plant parts may respond differently to abiotic stresses in terms of the secondary metabolite production. Using the concatenated LC-MS and NMR data, Sampaio et al. (2016) correlated the seasonal pattern of metabolite production in the leaf and stem of Tithonia diversifolia with the amount of rainfall and changes in temperature; however, metabolites in roots and inflorescences were affected mainly by some soil nutrients such as $\mathrm{Ca}, \mathrm{Mg}, \mathrm{P}, \mathrm{K}$ and $\mathrm{Cu}$. These metabolites mostly belong to the class of sesquiterpene lactones, flavonoids and trans-cinnamic-acid derivatives (mainly caffeoylquinic acid) and possess several therapeutic properties (Sampaio et al., 2016). The gaseous as well as heavy-metal pollutants are markedly hazardous when in high concentration. Application of $\mathrm{SO}_{2}$ stress at the seedling stage of Psoralea corylifolia reduced the psoralen contents of leaves, stem and roots, especially during the pre-flowering stage (Ali et al., 2008). Roots, although significantly affected like stem and leaves in the pre-flowering stage, showed a greater potential to resist during the flowering and post-flowering growth stages. $\mathrm{SO}_{2}$ effect was most visible in seeds, showing a drastic fall of psoralen content, i.e. from $5.32 \%$ in control to merely $0.71 \%$ in treated materials (Ali etal., 2008).

Similarly, ozone treatment for 62 days with a mean $\mathrm{O}_{3}$ concentration of $171.6 \mu \mathrm{g} \mathrm{m}^{3}$ in an open-top chamber caused a $50 \%$ decrease in the capsaicin level and about $53 \%$ increase in the total carotenoid content in the pericarp of the Capsicum baccatum fruit (Bortolin et al., 2016). In citrus plants, production of limonoids was altered by the drought and high temperature stress (Zandalinas et al., 2017).

The effect of coal-smoke pollutants was found to be positive on the hyoscyamine contents of roots, stem and leaves of Datura innoxia, which increased over the control by $67 \%$ in the stem, $109 \%$ in leaves and $1400 \%$ in roots of the field-grown plants. Interestingly, the increase was marginal and nonsignificant in the in-vitro-raised regenerants (Singh et al., 2000). Gamma irradiation (5-80 Gy) caused only insignificant changes in the hyoscyamine accumulation in this species, although low doses stimulated the metabolism, resulting in increased biomass production and photosynthetic efficiency (Aref et al., 2016). Application of $\mathrm{Zn}$ (a micronutrient) in low concentration quickened the process of hyoscyamine production as expected, but proved to be toxic in high concentration ( $\left.>400 \mu \mathrm{M} \mathrm{ZnSO}_{4}\right)$, causing a reduction in hyoscyamine content (Srivastava et al., 1993).

Seed oils of Ruellia tuberosa and Peristrophe bicalyculata, are known to exhibit tuberculostatic activity. The influence of coal-smoke pollution not only reduced the total oil content but also altered the relative proportion of the component fatty acids of these oils (Iqbal et al., 2011b). Of the caprylic acid (C8:0), capric acid (Cl0:0), lauric acid (Cl2:0) and myristic acid (Cl4:0) present in ruellia oil, the proportions of the first and the third FAs increased, while those of others decreased in the polluted samples, compared with the control. In peristrophe oil, the proportion of lignoceric acid (C24:0) declined from about 49 to 7 , while those of others (palmatic acid, stearic acid, arachidic acid and behenic acid) increased variously (Iqbal et al., 2011b). Similarly, salinity stress altered the FA proportions in the chicory (Cichorium intybus) seed oil; application of $\mathrm{NaCl}$ reduced the oil content and possibly disturbed the synthesis of long-chain FAs (Arshi et al., 2006a). It should not be surprising if such alterations 
in the ratio of fatty acids and other constituents of oils have a bearing on the therapeutic properties of these oils.

In a study by Qureshi et al. (2013), the fatty-acid profiling of Artemisia annua revealed a major salinity-induced shift towards the long-chain and mono-saturated fatty acids. Myristic acid (14:0), palmitoleic acid (16:1), linoleic acid (18:2) and erucic acid (22:1) increased by $141 \%, 186 \%, 34 \%$ and $908 \%$, respectively, in comparison with the control. The contents of oleic acid (18:1), linolenic acid (18:3), behenic acid (22:0) and lignoceric acid (24:0) decreased by $50 \%, 17 \%, 44 \%$ and $78 \%$, respectively. Modification in fatty-acid composition might be a membrane adaptation to the long-term salinity and oxidative stress. Since fatty acids are the building blocks of lipids, including membrane lipids, any change in lipid composition would also be crucial for plant adaptation to the stress applied (Qureshi et al., 2013).

The yields of essential oils of the peppermint (Mentha piperita) and apple mint ( $M$. suaveolens) plants were also reduced under salt stress. In pennyroyal (M. pulegium), on the contrary, salt stress increased the oil concentration in the tissue, suggesting that the process of oil synthesis/degradation was less sensitive to salt stress in this species (Aziz et al., 2008). Moreover, relative proportion of different constituents of the oil displayed varied responses. Similar experiments with Dracocephalum moldavica and Satureja hortensis brought out that salt stress as well as water stress causes alterations in the yield and composition of essential oils (Baher et al., 2002, Aziz and Taalab, 2004).

Sennoside, an anthracine derivative, which is laxative in action, is the main active principle of Cassia angustifolia plants. Its production was considerably reduced under salinity stress. The maximum decline, $64 \%$ and $60 \%$, was observed on application of $160 \mathrm{mM} \mathrm{NaCl}$ solution to the soil at pre-flowering and flowering stages, respectively. Application of $\mathrm{CaCl}_{2}$ caused an ameliorative effect and increased the sennoside content (up to 27\%) in comparison to the control (Arshi et al., 2006b). The total sennoside yield of a plant was dependent on the total biomass of pod and leaf (economic yield) and their sennoside content (Arshi et al., 2002, 2004, 2005).

Oxidative stress induced by heavy metals triggers signalling pathways, which regulate the production of specific metabolites. The reactive oxygen species (ROS) generated due to heavy-metal stress often causes lipid peroxidation, which facilitates formation of highly active signalling compounds capable of triggering the production of secondary metabolites (Nasim and Dhir, 2010). In a study of heavy metal stress on artemisinin accumulation in the leaves of Artemisia annua, lead stress dropped the artemisinin content from $464 \mathrm{\mu g} \mathrm{g}^{-1} \mathrm{dw}$ (control) to $226 \mu \mathrm{g} \mathrm{g}^{-1} \mathrm{dw}$ (with $100 \mathrm{ppm} \mathrm{Pb}$ ), $180 \mathrm{\mu g} \mathrm{g}^{-1}$ (with $250 \mathrm{ppm} \mathrm{Pb}$ ) and $173 \mu \mathrm{g} \mathrm{g}^{-1} \mathrm{dw}$ (with $500 \mathrm{ppm} \mathrm{Pb}$ ), showing a general decline of artemisinin content with increase in the level of $\mathrm{Pb}$ applied (Qureshi et al., 2005). A similar trend appeared for the per plant yield of artemisinin, which dropped from $16.09 \mathrm{mg}$ in the control to $6.56 \mathrm{mg}, 2.58 \mathrm{mg}$ and $1.54 \mathrm{mg}$ per plant at these three stress levels, respectively. A similar variation pattern of the content and total yield of artemisinin by was obtained by the application of salinity (NaCl) stress on Artemisia annua (Qureshi et al., 2005).

Plant growth regulators : The metabolite levels in tissues are also influenced by plant growth regulators. The percentage of alcohols and monoterpenes in Clary Sage (Salvia sclarea) generally declined with the application of kinetin, indole acetic acid (IAA) or paclobutrazol (PBZ). The level of sesquiterpene (Bcaryophyllene) gave a diverse response, showing an increase with kinetin, a decrease with IAA and an irregular and inconspicuous variation with $\mathrm{PBZ}$, as compared with the control. Of the acetates present, proportion of linaryl acetate increased, while those of neryl acetate and geranyl acetate declined with each of the PGR used (Singh et al., 2008). Application of PBZ maximally enhanced the oil yield and improved the linalool-linalylacetate content by about $12 \%$ over the control (Singh et al., 2008). Using the fungal species Aspergillus niger and Rhizopus oligosporus as biotic stress whereas salicylic acid and methyl jasmonate as abiotic stress, in vitro production of secondary metabolites in coffee bean (Coffea canephora) was enhanced by Vaddadi and Parvatam (2015). Abiotic stress was found to be more effective than the biotic stress in stimulating the production of caffeine, theobromine, cafestol, kahweol, trigonelline and its derivative nicotinic acid (Vaddadi and Parvatam, 2015).

The above discussion indicates that controversies regarding the botanical identity of herbal drugs can be resolved reliably by carefully estimating the contents of their active ingredients responsible for therapeutic impact of the given drug. However, since the quantity of such biocompounds is variable with numerous internal and external factors, analysis of (i) any one part or organ of the plant at any one stage of plant ontogeny, (ii) a single genotype of a given species, or (iii) a single plant population growing in a locality with specific climatic and environmental set up, cannot give an exact idea of the actual magnitude of the amounts of active ingredients produced. Therefore, several samples from varied agro-climatic conditions, collected at different stages of plant growth and covering the various plant parts/organs of the given species need to be analyzed to determine which of the given plant species should be considered as the actual drug. If a species has more than one genotypes, all of them should be scanned for arriving at a judicious and authentic conclusion.

Furthermore, environmental stress may affect not only the overall quantity of the desired active ingredients but sometimes even their composition and hence the quality may be affected. This may also influence the overall therapeutic efficacy of the drug. If this happens, revision of drug doses may also become essential to maintain the expected curative effect of the drug. On the whole, not only the quality, and efficacy of drugs, but also their sustainable availability has to be ensured in view of the rapidly-growing demand of herbal medicine all over the globe. 
Promotion of an organized cultivation of medicinal plants can be a right step in right direction.

\section{Conclusion}

Most of the criteria currently in practice to characterize plant drugs are ineffective. In cases where several plant species are in use under one vernacular name of drug, the best way to resolve the actual botanical identity of the drug and determine which of these different plant species is in fact the genuine drug is to estimate the active ingredients of all the species involved; the one with the maximum content of the concerned active principle should be regarded as actual drug, while the next in richness with this compound as the alternative drug. Those species poor in content of the desired ingredient may be rejected. However, since the amount of active ingredients of plants varies with numerous internal and external environmental factors, as pointed out in this commentary, effort should be made to take all possible precautions while quantifying these compounds of therapeutic importance.

\section{Acknowledgements}

Authors extend their appreciation to the International Scientific Partnership Program (ISPP) at King Saud University, Riyadh for funding this research work through ISPP \# 00113, and thank Prof. M.P. Sharma, Jamia Hamdard for his help with botanical nomenclature. We respectfully dedicate this work to esteemed Prof. S.N. Chaturvedi (formerly with RBS College, Agra) on his $90^{\text {th }}$ birth anniversary and pray for his long and healthy life.

\section{References}

Ahmad, W., S.M.A. Zaidi and S. Ahmad: Quality control analysis of Didymocarpus pedicellata R Br. Indian J. Trad. Knowl., 13, 175180 (2014).

Al-Gabbiesh, A., M. Kleinwächter and D. Selmar: Influencing the contents of secondary metabolites in spice and medicinal plants by deliberately applying drought stress during their cultivation. Jordan J. Biol. Sci., 8, 1-10 (2015).

Ali, M.B.: Secondary metabolites and environmental stress in plants: biosynthesis, regulation and function. In : Physiological Mechanisms and Adaptation Strategies in Plants under Changing Environment. (Eds. : P. Ahmad and M.R. Wani) Vol. 2. Springer Science + Business Media, New York, pp. 55-85 (2014).

Ali, S.T., Mahmooduzzafar, M.Z. Abdin and M. Iqbal: Ontogenetic changes in foliar features and psoralen content of Psoralea corylifolia Linn. exposed to $\mathrm{SO}_{2}$ stress. J. Environ. Biol., 29, 661668 (2008).

Anis, M., M.P. Sharma and M. Iqbal: M, Herbal ethnomedicine of the Gwalior forest division in Madhya Pradesh, India. Pharmaceut. Biol., 38, 241-253 (2000).

Anonymous: Plant World 2000. Herbal Bull., 3, 1-5 (2000).

Anonymous: Ethnobiology in India: A Status Report. Ministry of Environment \& Forests, Govt. of India, New Delhi, India (1994).

Anonymous: The Unani Pharmacopoeia of India, CCRUM, Department of AYUSH, Ministry of Health and Family Welfare, Govt of India, New Delhi (2007).
Aref, I.M., P.R. Khan, A.A. Al-Sahli, A. Husen, M.K.A. Ansari, Mahmooduzzafar and M. Iqbal: Response of Datura innoxia Linn. to gamma rays and its impact on plant growth and productivity. Proc. Nat. Acad. Sci. India (B), 86, 623-629 (2016).

Arshi, A., M.Z. Abdin and M. Iqbal : Growth and metabolism of senna as affected by salt stress. Biol. Plant., 45, 295-298 (2002).

Arshi, A., M.Z. Abdin and M. Iqbal : Changes in biochemical status and growth performance of senna (Cassia angustifolia Vahl.) grown under salt stress. Phytomorphology, 54, 109-124 (2004).

Arshi, A., M.Z. Abdin and M. Ibqal: Ameliorative effect of $\mathrm{CaCl}_{2}$ on growth, ionic relations and proline content of senna under salinity stress. J. Plant Nutr., 28, 101-125(2005).

Arshi, A., M.Z. Abdin and M. Iqbal: Effects of $\mathrm{CaCl}_{2}$ on growth performance, photosynthetic efficiency and nitrogen assimilation of Cichorium intybus L. grown under $\mathrm{NaCl}$ stress. Acta Physiol. Plant., 28, 137-147 (2006a).

Arshi, A., M.Z. Abdin and M. Iqbal: Sennoside content and yield attributes of Cassia angustifolia Vahl. as affected by $\mathrm{NaCl}$ and $\mathrm{CaCl}_{2}$. Sci. Hort., 111, 84-90 (2006b).

Aziz, E.E., H. Al-Amier and L.E. Craker: Influence of salt stress on growth and essential oil production in peppermint, pennyroyal and apple mint. J. Herbs Spices Med. Plants., 14, 77-87 (2008).

Aziz, E.E. and S.M. Taalab: Dragonhead plants (Dracocephalum moldavica) responses to salt stress and different sources of sulphur. Egyptian J. Appl. Sci., 19, 239-257 (2004).

Aziz, Z.A., M.R. Davey, J.B. Power, P. Anthony, R.M. Smith and K.C. Lowe: Production of asiaticoside and madecassoside in Centella asiatica in vitro and in vivo. Biol. Plant., 51, 34-42 (2007).

Baher, L., F. Zahra, M. Mirza, M. Ghorbanli and M.B. Rezaii: The influence of water stress on plant height, herbal and essential oil yield and composition in Satureja hortensis. Flav. Fragr., 17, 275277 (2002).

Beigh, S.Y., I.A. Nawchoo and M. Iqbal: Herbal drugs in India: Past and present uses. J. Trop. Med. Plants, 3, 197-204 (2002).

Beigh, S.Y., I.A. Nawchoo and M. Iqbal: Traditional veterinary medicine among the tribes of Kashmir Himalaya. J. Herbs Spices Med. Plants, 10, 121-127 (2003).

Beigh, S.Y., I.A. Nawchoo and M. Iqbal: Cultivation and conservation of Aconitum heterophyllum: Acritically endangered medicinal herb of the north-west Himalaya. J. Herbs Spices Med. Plants, 11, 47-56 (2005).

Beigh, S.Y., I.A. Nawchoo and M. Iqbal : Alkaloids contents of Aconitum heterophyllum growing at different altitudes of the Kashmir Himalaya. J. Indian Bot. Soc., 87, 252-255 (2008).

Beigh, S.Y., I.A. Nawchoo, M.P. Sharma and M. Iqbal: Traditional herbal therapy in the Kashmir Himalaya. In: Current Trends in Medicinal Botany (Eds. : M. Iqbal and A. Ahmad). IK International, New Delhi, pp. 1-14 (2014).

Bortolin, R.C., F.F., Caregnato, A.M.D., Junior, A., Zanotto-Filho, K.S., Moresco, A., de Oliveira Rios, D.P. Gelain: Chronic ozone exposure alters the secondary metabolite profile, antioxidant potential, antiinflammatory property, and quality of red pepper fruit from Capsicum baccatum. Ecotox. Environ. Saf., 129, 16-24 (2016).

Chan, K.L., K.H. Yuen, S. Jinadasa, K.K. Peh and W.T. Toh: A high performance liquid chromatography analysis of plasma artemisinin using a glassy carbon electrode for reductive electrochemical detection. Planta Med., 83, 68-69 (1997).

Chaudhary, A.A., A. Ahmad and M. Iqbal: Molecular biology techniques for authentication of medicinal plants. In: Current Trends in Medicinal Botany (Eds. : M. Iqbal and A. Ahmad), IK International, New Delhi, pp. 311-345 (2014).

Cordell, G.A. : Ecopharmacognosy and the globalization of traditional 
medicines. Indian J. Trad. Knowl., 14, 595-604 (2015).

Dar, G.H. and S. Farooq: How diverse is biodiversity! Do we know? Oriental Sci., 2, 51-69 (1997).

Datta, A. and P.S. Srivastava: Variation in vinblastine production of Catharanthus roseus during in vivo and in vitro differentiation. Phytochemistry, 46, 135-137 (1997).

Dhir, B.: Environmental Toxicity and Alterations in Medicinal Plants. Nova Science Publishers, Inc., New York (2015).

Dong, J. E., X.H. Ma, Q. Wei, S.B. Peng and S.C. Zhang: Effects of growing location on the contents of secondary metabolites in the leaves of four selected superior clones of Eucommia ulmoides. Ind. Crop Prod., 34, 1607-1614 (2011).

Dorais, M., A.P. Papadopulos, X. Luo, S. Leonhart, A. Gosselin, K. Pedneault, P. Angers and L. Gaudreau: Soil-less greenhouse production of medicinal plants in northeastern Canada. Acta Hort., 554, 186-189 (2001).

Farooqi, A.H.A. and S. Haque: Secondary metabolite production of medicinal and aromatic plants as influenced by nutrients and heavy metals. In : Medicinal Plants in Changing Environment (Eds. : A. Ahmad, T.O. Siddiqi and M. Iqbal). Capital Publishing Co., New Delhi, pp. 35-49 (2011).

Ferreira, J.F.S., J.E. Simon and J. Janick : Relationship of artemisinin content of tissue cultured, green house and field grown plants of Artemisia annua. Planta Med., 61, 351-355 (1995).

Garg, M., E.T. Tamboli and S. Ahmad: Pharmacognostical evaluation of plant drugs with special emphasis on chromatography : Adhatoda vasica leaves. In : Current Trends in Medicinal Botany (Eds. : M. Iqbal and A. Ahmad) IK International, New Delhi, pp. 158-171 (2014).

Gulati, A., S. Bharel, S.K. Jain, M.Z. Abdin and P.S. Srivastava: In vitro micropropagation and flowering in Artemisia annua. J. Plant Biochem. Biotechnol., 5, 31-35 (1996).

Gulati, A., S.K. Jain and P.S. Srivastava: Experimental studies on Thevetia neriifolia Juss - A review. Indian J. Chem., 398, 808-812 (2000).

Hayden, A.: Aeroponic and hydroponic systems for medicinal herb, rhizome, and root crops. Hort. Sci., 41, 536--538 (2006).

lqbal, $\mathrm{M}$ : Adream has come true. In :A role model for leaders of change in India : Hakeem Abdul Hameed as Seen by His Contemporaries (Ed: A.R. Bedar). Educational Publishing House, Delhi, India, pp. 104-117 (2011).

Iqbal. M.: From medicine to phytomedicine. Ann. Phytomed., 2, 1-2 (2013).

Iqbal, M. and P.S. Srivastava : Some aspects of medicinal plant research that merit attention. In : Compendium on Phytomedicines (Ed.: D. Sharma). Council for Development of Rural Areas, CSIR Publications, New Delhi, pp. 424-436 (1998).

Iqbal, M., S.Y. Beigh and I.A. Nawchoo : Variability in morphology and active constituents of Podophyllum hexandrum, a Himalayan herb known for its anti-cancer activity. J. Trop. Med. Plants, 5, 33-36 (2004).

Iqbal, M., A. Ahmad and T.O. Siddiqi : Characterization of controversial plant drugs and effect of changing environment on active ingredients. In: Medicinal Plants in Changing Environment (Eds. : A. Ahmad, T.O. Siddiqi and M. lqbal). Capital Publishing Co, New Delhi, pp. 1-10 (2011a).

Iqbal, M., Mahmooduzzafar, F. Nighat and I.M. Aref: Composition of seed oils of Peristrophe bicalyculata and Ruellia tuberosa as affected by coal-smoke stress. J. Food Agric. Environ., 9, 1101-1104 (2011b).

Jain, S.K.: Global resurgence of ethnomedicobotany. The Indian scene. J. Trop. Med. Plants, 1, 75-81 (2000).

Katare, D.P. and M. Bora: Environmental stress enhances the level of secondary metabolites in medicinal plants. In: Medicinal Plants in Changing Environment (Eds. : A. Ahmad, T.O. Siddiqi and M. lqbal), Capital Publishing Co, New Delhi, pp. 98-108 (2011).

Khan, S. : Authentication of medicinal plants : Phyllanthus amarus. In: Medicinal Plants in Changing Environment (Eds. : A. Ahmad, T.O. Siddiqi and M. Iqbal). Capital Publishing Co, New Delhi, pp. 224248 (2011).

Klayman, D.L., A.J. Lin, N. Acton, J.P. Scovill, J.M. Hock and W.K. Milhous: Isolation of artemisinin (qinghaosu) from Artemisia annua growing in the United States. J. Nat. Prod., 47, 715-717 (1984).

Kliebenstein, D.J.: Secondary metabolites and plant/environment interactions: a view through Arabidopsis thaliana tinged glasses. Plant Cell Environ., 27, 675-684 (2004).

Krishnamurthy, K.H. and G.C. Mouli: Siddha system of medicine: A historical appraisal. Indian J. Hist. Sci., 19, 43-53 (1984).

Liersch, R., H. Soicke, C. Stehr and H.U. Tullner: Formation of artemisinin in Artemisia annua during one vegetation period. Planta Med., 52, 387-390 (1986).

Liu, W., J. Liu, D. Yin and X. Zhao: Influence of ecological factors on the production of active substances in the anticancer plant Sinopodophyllum hexandrum (Royle). PLOS One, 10, e0122981, (DOI: 10.1371/journal), pp 22 (2015).

Liu, W., D. Yin, N. Li, X. Hou, D. Wang, D. Li and J. Liu: Influence of environmental factors on the active substance production and antioxidant activity in Potentilla fruticosa L. and its quality assessment. Sci. Rep., 6, 28591 (DOI: 10.1038/srep28591), 18 (2016).

Luo, H.M., P.P. Chao, C.C. Yu, C.V. Tai and C.W. Liu: TLC scanning determination of artemisinin in Artemisia annua. Chin. Pharm. Bull., 15, 8-10 (1980).

Mohsin, M., A. Ahmad and M. Iqbal: FRET-based genetically encoded sensors for quantitative monitoring of metabolites. Biotechnol. Lett., 37, 1919-1928 (2015).

Mughal, M.H., G. Ali, P.S. Srivastava and M. Iqbal: Improvement of drumstick (Moringa pterygosperma Gaertn.), a unique source of food and medicine, through tissue culture. Hamdard Med., 42, 3742 (1999)

Nasim, S.A. and B. Dhir: Heavy metals alter the potency of medicinal plants. Rev. Environ. Contam. Toxicol., 203, 139-149 (2010).

Pagilarulo, C.L., A.L. Hayden and G.A. Giacomelli : Potential for greenhouse aeroponic cultivation of Urtica dioica. Acta Hort., 659, 61-69 (2005).

Pasquale, A.D.: Pharmacognosy: The oldest modern science. J. Ethnopharmacol., 11, 1-16 (1984).

Purohit, M., S. Pande, A. Datta and P.S. Srivastava: In vitro flowering and high xanthotoxin in Ammi majus. Plant Biochem. Biotechnol., 47376 (1995a).

Purohit, M., S. Pande and P.S. Srivastava: Enhanced xanthotoxin content in regenerating cultures of Ammi majus and micropropagation. Planta Med., 61, 481-482 (1995b).

Qureshi, M.I., M. Israr, M.Z. Abdin and M. Iqbal: Responses of Artemisia annua L. to lead and salt-induced oxidative stress. Environ. Exp. Bot., 53, 185-193 (2005).

Qureshi, M.I., M.Z. Abdin, J. Ahmad and M. Iqbal: Effect of long-term salinity on cellular antioxidants, compatible solute and fatty acid profile of sweet annie (Artemisia annua L.). Phytochemistry, 95, 215-223 (2013).

Radušiene, J., B. Karpavičienè, and Z. Staniun: Effect of external and internal factors on secondary metabolites accumulation in St John's worth. Bot. Lith., 18, 101-108 (2012).

Raghunathan, K. : Medico-ethnobotanical surveys and their role in research in Ayurveda. A Manual of Ethnobotany, (Eds. : S.K. Jain) 
Scientific Publishers, Jodhpur, India, pp.79-93 (1987).

Ramakrishna, A. and G.A. Ravishankar: Influence of abiotic stress signals on secondary metabolites in plants. Plant Signal Behav., 6, 1720-1731 (2012).

Sampaio, B.L., R.A. Edrad-Ebel and F.B. Da Costa: Effect of the environment on the secondary metabolic profile of Tithonia diversifolia: A modal of environmental metabolomics of plants. Sci. Reports, 6, 29265 (2016). (DOI: 10.1038/srep 29265)

Sarwat, M. and G. Nabi: Amplified fragment length polymorphism: A useful and versatile technique for medicinal plant research. In: Current Trends in Medicinal Botany, (Eds. : M. Iqbal and A. Ahmad) IK International, New Delhi, pp. 288-310 (2014).

Selmar, D. and M. Kleinwächter : Stress enhances the synthesis of secondary plant products : The impact of stress-related overreduction on the accumulation of natural products. Plant Cell Physiol., 54, 817-826 (2013).

Shane, S. : Science of life: A brief history of Ayurvedic medicine from mythology to modern day. The Mindful Word. Newsletter (2014).

Singh, A., V.K. Kaul, V.P. Mahajan, A. Singh, L.N. Misra and R.S. Thakur: Introduction of Artemisia annua in India and isolation of artemisinin, a promising antimalarial drug. Indian J. Pharmaceut. Sci., 48, 137-138 (1986).

Singh, N., G. Ali, W.Y. Soh and M. Iqbal: Growth responses and hyoscyamine content in Datura innoxia under the influence of coalsmoke pollution. J. PlantBiol., 43, 69-75 (2000).
Singh, V., R. Sood, K. Ramesh and B. Singh: Effect of growth regulator application on growth, flower, oil yield and quality of Clary page (Salvia sclarea L.). J. Herbs Spices Med. Plants, 14, 29-36 (2008).

Srivastava, P.S., M. Purohit, D. Pande and A. Datta: Phenotypic variation and alkaloid content in androgenic plantlets of Datura innoxia. Phytomorphology, 43, 209-216 (1993).

Stavroula, M. and J. Rahul: Mediterranean climate affects the biosynthesis of secondary metabolites in common medicinal plants. Int. J. Bot., 6, 17-28 (2017).

Steven, P. F.: Angiosperm Phylogeny Website (http://www.mobot.org /MOBOT/research/APweb/.) Version 12 (2012).

Swamy, B.G.L.: The date of Tholkappium - a retrospect. Ann. Orient. Res., 25, 292-315(1975).

Tabatabaei, S.J.: Effect of cultivation systems on the growth and essential oil contents and composition of Valerian. J. Herbs Spices Med. Plants, 14, 54-67 (2008).

Vaddadi, S. and G., Parvatam : Impacts of biotic and abiotic stress on major quality-attributing metabolites of coffee beans. J. Environ. Biol., 36, 377-382, (2015).

Zandalinas, S.I., C. Sales, J. Beltran, A. Gomez-Cadenas and V. Arbona: Activation of secondary metabolism in citrus plants is associated to sensitivity to combined drought and high temperatures. Front. Plant Sci., 7, 1954 (2017). (doi: 10.3389/fpls.2016.01954).

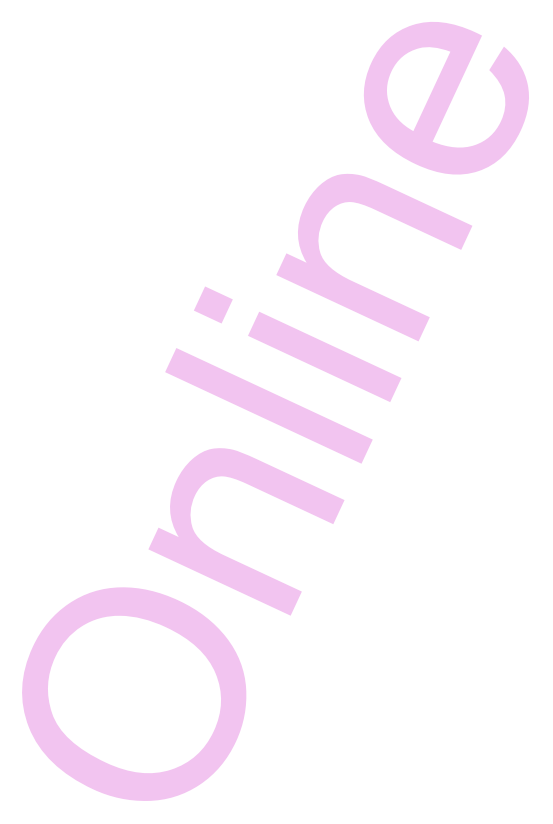

